\title{
THE GEOMETRIZATION OF GALILEI'S PRINCIPLE CHRISTIAN GÜNTHER
}

Dept. of Mathematics

Univerity of Rochester

Rochester, N.Y. 14627

\begin{abstract}
Galilei's fundamental principle is reformulated in terms of abstract geometry and it is generalized to nonlinear and relativistic systems. As a consequence the notion of a general mechanical system is developed and shown to be a universal frame for particle dynamics.
\end{abstract}

Keywords: Galilei's principle, symplectic geometry, evolution space, gauge theory.

AMS Subject Classification: 58F05, 58E30, 70-02,70B05, 70D05.

\section{Kinematics and Space-time geometry}

Modern (post Aristotelian) physics rests on Galilei's fundamental principle

Free bodies move on straight lines with constant velocity.

In this principle several assumptions about space and time (most still from Aristotelian physics) are made:

0 ) The motion of objects is described by curves $c(t)$ in space $Q$.

1)The space $Q$ is a 3-dimensional affine space with Euclidean structure.

Under these assumptions Galilei's principle can be reformulated using calculus:

The motion of free objects is described by curves $c(t)$ in $E^{3}$ given by the equation $c^{\prime \prime}(t)=0$. 
As an immediate consequence one obtains: The trajectory of a free particle is uniquely determined by the initial conditions: $c\left(t_{0}\right)=x_{O}$ and $c^{\prime}\left(t_{O}\right)=y_{O}$, i.e. by elements of the tangent bundle $\mathrm{TE}^{3}$.Therefore, trajectories are determined by second order differential equations on $\mathrm{Q}$ or in geometric language: The trajectories are the geodesics of the natural flat connection on the affine space $\mathrm{E}^{3}$.

In the course of history of mechanics these assumptions were modified several times in order to treat more complex situations such as systems of particles, systems with constraints, rigid bodies, and other nonlinear systems. The notion of space was generalized without changing the essential geometric contents of the original concept. The affine linear space is replaced by the more abstract concept of the configuration space and the lines are replaced by the geodesics of a connection $\nabla$. Thus the assumptions above mean:

0 ) The motion of a mechanical system is described by curves $c(t)$ in the configuration space $\mathrm{Q}$.

$\left.1^{\prime}\right) \mathrm{Q}$ is a n-dimensional differentiable manifold with a connection $\nabla$, the inertial connection of the system.

\section{Comments:}

Physically $\nabla$ represents the choice of a class of inertial systems.

Without a connection it would not be possible to define the acceleration $\nabla_{\mathrm{C}^{\prime}} \mathrm{c}^{\prime}$ of an object. Galilei's principle can now be reformulated for classical mechanical systems with a nonlinear configuration space Q:

\section{The trajectories of free particles are $\nabla$-geodesics,} i.e. curves $c(t)$ on the configuration space $Q$ with $\nabla_{c^{\prime} c^{\prime}}=0$.

A second fundamental principle of post Aristotelian physics is the principle of relativity which states the physical equivalence of all inertial systems. A mathematical formulation of this principle requires the concept of configuration space-time, which adds a time direction to the configuration space: 
Definition: A general configuration space-time $S$ is a differentiable manifold together with a connection $\nabla . \nabla$ represents the class of admitted inertial systems.

Comment: $\mathrm{S}$ represents the positions and time of all possible events. Thus $\mathrm{S}$ can also be called the event space.

Since the notion of space-time includes time as a coordinate, the speed of a trajectory is given by the direction and not the lengh of the tangent vector. Therefore, for all mechanical systems (including relativistic and quantum mechanics as will be shown below), Galilei's principle can be formulated using space-time:

\section{The trajectories of free systems are unparametrized $\nabla$-geodesic paths in configuration space-time (i.e a one dimensional family of events).}

\section{Causality:}

In addition to the inertial connection every system has a causal structure, which is determined by the type of signals admitted for measurements. In Galilean mechanics one assumes the existence of an absolute time, as universal parametrization for the trajectories.

Definition: A nonrelativistic configuration space-time is a 4-dimensional manifold $\mathrm{M}$ together with a connection $\nabla$, and a function $t: M \rightarrow \mathbb{R}$, the time, such that $(M, t)$ is a fibre bundle over $\mathbb{R}$.

Example: The configuration space-time of a particle in $\mathrm{Q}$ is $\mathbb{R Q}$. For $\mathrm{Q}=\mathrm{E}^{3}$ one obtains the nonrelativistic space-time $\mathrm{Q} \times \mathrm{E}^{3} \cong \mathbb{R}^{4}$.

Postulate: Trajectories of a nonrelativistic particle system are curves $c(t)$ in $M$ with $t^{\circ} \mathrm{c}=1_{M}$ (i.e.sections of $t$ ). Thus the trajectories are parametrized by the universal time.

In relativity these assumptions on space-time are replaced by:

Definition: A relativistic configuration space-time is a manifold $\mathrm{S}$ together with a cone bundle CS on its tangent bundle, which is induced by a pseudoriemannian metric $\mathrm{g}$ of signature $(1,3)$ such that $\nabla$ is a Pseudoriemmanian connection.

Definition: A tangent vector $\mathrm{v}$ is called

timelike iff $\mathrm{v} \in \mathrm{CS}$ (i.e. $\mathrm{g}(\mathrm{v}, \mathrm{v})>0$ ) 
lightlike iff $\mathrm{v} \in \partial \mathrm{CS}$ (i.e. $\mathrm{g}(\mathrm{v}, \mathrm{v})=0$ )

spacelike otherwise (i.e. $\mathrm{g}(\mathrm{v}, \mathrm{v})<0$ )

Remark: In nonrelativistic configuration, space-time is $\mathrm{CS}:=\{\mathrm{v} \in \mathrm{TS} \mid \mathrm{dt}(\mathrm{v})>0\}$, which is also a cone structure. Here $\mathrm{v}$ is timelike iff $\operatorname{dt}(\mathrm{v}) \neq 0, \mathrm{v}$ is lightlike iff it is spacelike iff $\mathrm{dt}(\mathrm{v})=0$.(This reflects the assumption that in nonrelativistic mechanics lightlike signals have "infinite" speed.)Thus one has:

A causal structure on a configuration space-time is a cone bundle CS $\subset$ TS. Remark: CS is determined by the type (finite or infinite speed) of signals used to examine space-time)

For relativistic space-times the cone structure isequivalent to a conformal structure. This structure is induced by a class of pseudoriemannian metrics on $\mathrm{S}$ which differ only by a function factor. Thus a conformal structure determines a family of lightlike geodesics. A connection $\nabla$ is called compatible iff

1) $\nabla$ is a conformal connection and

2) every lightlike geodesic of the cone structure is also a $\nabla$-geodesic. Thus a relativistic space-time is a Weyl-manifold [4].

For nonrelativistic space-times the cones $\mathrm{C}$ are degenerate and induced by $\mathrm{dt} \otimes \mathrm{dt}$. A connection is compatible iff

1. $\nabla d t=0$. In that case its geodesics $c(r)$ are linear in $t$ i.e. $t(c(r))=a r+b$, i.e. $c$ is up to a linear function parametrized by $t$.

2. The connection is assumed to be a Riemannian connection for the metric $\mathrm{k}$ on each fibre of S i.e. on the spacelike surfaces. This implies that the lightlike geodesics are the shortest lines in the configuration spaces $\mathrm{Q}$, which recovers the original (time independent) formulation of Galilei's priciple. Such nonrelativistic space-times will be called Galilei manifolds .

Scales: The choice of a cone base, i.e. a subset $B S \subset C S$ such that $C S=\mathbb{R} \times B S$, is physically the choice of a scale.

For relativistic systems the choice of a scale is equivalent to the choice of a particular $\mathrm{g}$ of the conformal class such that $\mathrm{BS}=\{\mathrm{v} \in \mathrm{TS} \mid \mathrm{g}(\mathrm{v}, \mathrm{v})=1\}$. 
For nonrelativistic systems a scale BS determines dt in its conformal class and $\mathrm{BS}=\{\mathrm{v} \in \mathrm{TS} \mid \mathrm{dt}(\mathrm{v})=0\}$.

Thus for all systems, relativistic as well as nonrelativistic one has :

The configuration space-time is a differentiable manifold $S$ together with a cone bundle CS $\subset$ TS (the causal structure), a choice of scale, a Riemannian structure on all spacelike hypersurfaces and a compatible connection $\nabla$.

\section{Covariance and inertial systems:}

Definition: An inertial system is physically the choice of a rest system at every point in space-time, and is geometrically the choice of a spacelike fibration in space-time.

Remark: For nonrelativistic systems a space like fibration of space-time $S$ is equivalent to a connection for the bundle $S \rightarrow \mathbb{R}$.

Since this fibration is transversal to the time fibration a local product structure on $\mathrm{TS}=\mathrm{VerS} \oplus$ HorS is induced and one obtains a metric $\mathrm{g}=\mathrm{k}+\mathrm{dt} \otimes \mathrm{dt}$ on $\mathrm{S}$. But note that $\mathrm{g}$ depends on the choice of an inertial system.

For relativistic space-times a space-like fibration is, via the g-orthogonal complement, equivalent with the choice of a time-direction at every point of S. Again one has a local splitting into space and time.

The principle of relativity states independence of physical laws of such a choice.

Definition: Propositions about systems or objects defined on configuration space-time will be called covariant if they depend only on the causal cone CS, the inertial connection, and the spacelike metric but not on the choice of an inertial system.

Example: The metric $\mathrm{g}$ on $\mathrm{S}$ is covariant for relativistic systems but not for nonrelativistic systems.

Remark: A proposition or an object is covariant iff it is invariant under the automorphism group of configuration space-time (S,PS, $\nabla$ ). For the classical nonrelativistic space-time $\mathbb{R} \times \mathbb{R}^{3}$ the automorphism group is the Galilei group, for Minkowsi space it is the Poincare group.

Thus one can formulate the principle of relativity:

The laws of physics are covariant. 
Initial conditions: In both the relativistic and the nonrelativistic case a free trajectory is uniquely determined by a point $\mathrm{x}$ in $\mathrm{S}$ and a tangent direction $\mathbb{R v}$ (a ray) (or for oriented trajectories a halfray $\mathbb{R}+v)$. Therefore, the space of initial conditions for free trajectories is the bundle of rays (resp. halfrays for oriented trajectories) PS:=(TS $\backslash$ zero section)/(R $\backslash\{0\}$ ) (resp. $\mathrm{P}^{+} \mathrm{S}=\left(\mathrm{TS} \backslash z \mathrm{zero}\right.$ section) $/ \mathbb{R}^{+}$) i.e. a bundle of projective spaces (resp. spheres).

By the definition of a cone base there is a covariant diffeomorphism between BS and PS+, the space of all timelike rays.

For nonrelativistic systems this map is given by: $\mathbb{R v} \mapsto v / d t(v)$. and, therefore, the space of all initial conditions is under the choice of an inertial system isomorphic with VerS or, if $S=\mathbb{R Q}$, with $\mathbb{R} \times \mathrm{TQ}$.

For relativistic systems this map is simply given by $\mathrm{v} \mapsto\left\|_{\mathrm{V}}\right\|$ thus all initial conditions are in the unit sphere of $\mathrm{g}$.

Parametrizations: In a nonrelativistic space-time there is a one-to-one correspondence between unparametrized timelike geodesic paths (i.e. trajectories of free systems) and geodesics $\mathrm{c}(\mathrm{t})$ parametrized by $\mathrm{dt}\left(\mathrm{c}^{\prime}(\mathrm{t})\right)=1$.

For relativistic systems there is no such natural parameter, but the timelike geodesics can be parametrized by $\mathrm{g}\left(\mathrm{c}^{\prime}(\mathrm{t}), \mathrm{c}^{\prime}(\mathrm{t})\right)=1$, the proper time parametrization.

For nonrelativistic systems one has a map PS\{lightlike rays $\} \rightarrow\{v \in T S \mid$ $\mathrm{dt}(\mathrm{v})=1\}$.This affine bundle is the space of initial conditions for all trajectories parametrized by the time $t$.

For relativistic systems the space of initial conditions for oriented timelike geodesics can be identified with the $g$-spherebundle $\{v \in T S \mid g(v, v)=1\}$. Finally the geodesics in $S$ can also be expressed as the extremals of a variational problem:

Theorem: The trajectories of free systems in a configuration space-time $S$ are the extremals of the variational problem: 


$$
\delta \int_{t_{1}}^{t_{2}}\left\|c^{\prime}(t)\right\| d t=0
$$

Note: The integrand is a covariant function only for relativistic systems. For nonrelativistic systems a choice of inertial system is needed to obtain a metric and thus a norm on TS.

Remark: The integrand is a positively homogeneous function of degree one. This is because of the following proposition:

Proposition: [10] The integrand of a variational problem is (positively) homogeneous of degree one if and only if with every extremal $c(t)$ also every (orientation preserving) reparametrization is an extremal, i.e. the extremals can be interpreted as unparametrized (oriented) paths.

\section{Newton's law and presvmplectic dynamics:}

Newtons law for a particle in $\mathrm{E}^{3}$ is usually formulated as follows

$$
F(t, c(t))=m^{\prime \prime}(t) \text {. }
$$

This introduces forces as a description of the deviation of a trajectory from the free case. Thus Newton's law is a logical consequence of Galilei's principle. The novelty of Newtons concept of force (as opposed to Aristoteles) is the fact that $\mathrm{F}$ is not necessarily attached to an object, $\mathrm{F}$ is a function of time and space, i.e. a field.

The constant $\mathrm{m}$, the mass, is a characteristic for the system. Given the force $\mathrm{F}$ Newton's law is a second order differential equation for the trajectories of a system. Systems with the same mass and same initial conditions in PS will have the same trajectories.

For general configuration spaces the acceleration of a trajectory is $\nabla_{\mathrm{c}^{\prime}} \mathrm{c}^{\prime}$, thus Newtons law can be written as $F=m \nabla_{c^{\prime}} c^{\prime}$. But in order to incorporate the mass $m$ into the space of initial conditions the concept of momentum and evolution space will be developed in the following.

Momenta and Evolution space; Let $h$ be either $g$ or $d t \otimes d t$ i.e. the form which determines the cone $C S$ and its base BS. For a system with configuration space-time $S$ the metric $g$ induces a natural vector bundle isomorphism $\mathrm{g}^{\mathrm{b}}: \mathrm{T}^{*} \mathrm{~S} \rightarrow \mathrm{TS}$ given by $\mathrm{g}^{\mathrm{b}}(\mathrm{v})=\mathrm{g}(\mathrm{v}, \cdot)$. Note that $\mathrm{g}^{\mathrm{b}}$ is covariant only for relativistic systems. Define the bundle map $\Phi: \mathrm{PS}^{+} \rightarrow \mathrm{T}^{*} \mathrm{~S}$ 
given by $\mathrm{v} \rightarrow \mathrm{g}(\mathrm{v}, \cdot) /\left\|_{\mathrm{v}}\right\|_{\mathrm{h}} . \Phi$ is one-to-one and the image of $\Phi$ is in $\mathrm{B}^{*} \mathrm{~S} \subset \mathrm{C}^{*} \mathrm{~S}$, where $\mathrm{C}^{*} \mathrm{~S}=\mathrm{g}^{\mathrm{b}}(\mathrm{CS})$ and $\mathrm{B}^{*} \mathrm{~S}=\mathrm{g}^{\mathrm{b}}(\mathrm{BS})$, the induced cone and cone base in $\mathrm{T}^{*} \mathrm{~S}$.

For nonrelativistic systems one has image $\Phi=\left\{\alpha \in T^{*} S \mid \alpha\left(\frac{\partial}{\partial t}\right)=1\right\}$, where $\frac{\partial}{\partial t}$ is the dual of $\mathrm{dt}$ with respect to $\mathrm{g}$ and thus dependent on the choice of an inertial system. While $\Phi$ is not covariant for nonrelativistic systems, any inertial system induces isomorphisms $\mathrm{BS} \rightarrow \operatorname{VerS}$ and $\mathrm{B}^{*} \mathrm{~S} \rightarrow \operatorname{Ver}^{*} \mathrm{~S}$ and these isomorphisms are independent of the inertial system, i.e. they are covariant. $\Phi$ is covariant for relativistic systems.

Definition: The evolution space of a system with mass $m$ is $E:=\left\{p \in T^{*} S \mid h^{*}(p, p)=m^{2}\right\}$, the closure of image $\Phi$. Elements of $\mathrm{E}$ are the momenta of the system.

As a codim 1 submanifold of $T^{*} S, E$ is a presymplectic manifold $(E, \omega)$, with $\omega=-d \vartheta$, where $\vartheta$ is the fundamental one-form. Moreover $\operatorname{dimKer} \omega=1$.

Definition: The map $\mathbb{F L}_{0}: \mathrm{TS} \rightarrow \mathrm{E}, \mathbb{F L}_{0}(\mathrm{v})=\mathrm{mg}(\mathrm{v}, \cdot) /\left\|_{\mathrm{v}}\right\|_{\mathrm{h}}, \mathrm{m} \in \mathbb{R}$ is called the Legendretransformation.

For a nonrelativistic particle with configuration space $\mathrm{Q}$ and configuration space-time $\mathbb{R} \times \mathrm{Q}$ the evolution space is isomorphic with $\mathrm{E}=\mathbb{R} \times T^{*} \mathrm{Q}$. The Legendre transformation can then be written as the map $T^{*}(\mathbb{R Q}) \rightarrow \mathbb{R} \times T^{*} Q,(t, v) \mapsto$ mv the momentum of the system if a rest system is chosen. The change of the choice of inertial system induces an affine and thus presymplectic diffeomorphism on $\mathrm{E}$.

For relativistic particles the Legendre transformation is simply $\mathrm{v} \mapsto \mathrm{g} b(\mathrm{v}, \cdot) /\left\|_{\mathrm{v}}\right\|$. In the following, the relativistic case will be restricted to a single particle because of the known problems with relativistic interaction of particles.

Definition: Denote by $\mathbb{F}$ the fibrederivative along the fibres of TS. The function $\mathrm{F}: \mathrm{TS} \rightarrow \mathbb{F L}: \mathrm{TS} \rightarrow \mathrm{T}^{*} \mathrm{~S}$ is the Legendretransformation of $\mathrm{L}$. $\mathrm{L}$ is called the Lagrangian.

Note: In the nonrelativistic case one obtains the Legendretransformation constructed above from the Lagrangian $\mathrm{L}_{0}: \mathrm{TS} \rightarrow \mathbb{R}, \mathrm{L}_{0}(\mathrm{~V})=\frac{1}{2} \mathrm{mv}^{2}$. In the relativistic case one has $\mathrm{L}_{0}(\mathrm{v})=\mathrm{m}\left\|_{\mathrm{v}}\right\|_{\mathrm{g}}$.

In both cases the trajectories of the free systems are extremals of the variational problem with Lagrangian $\mathrm{L}_{0}$ : 


$$
\delta \int_{\tau_{1}}^{\tau_{2}} L_{0}\left(c(\tau), c^{\prime}(\tau)\right) d \tau=0
$$

Let $\omega$ be the canonical symplectic form on the cotangent bundle $T^{*} S$ and let $\omega_{0}:=\mathbb{F} L_{0}{ }^{*} \omega$ be the pull back to TS. Furthermore let $\mathrm{A}: \mathrm{TS} \rightarrow \mathbb{R}$ be given by $\mathrm{A}(\mathrm{v})=\mathbb{F} \mathrm{L}_{0}(\mathrm{v})(\mathrm{v})$, the action, and let $\mathrm{E}:=\mathrm{A}-\mathrm{L}$ be the energy of the system. Then one has:

Proposition: A curve $\mathrm{c}(\tau)$ in $S$ is an extremal of the variational problem if and only if

$$
\left.c^{\prime \prime}(\tau)\right\lrcorner \omega_{0}=d E .
$$

In the nonrelativistic case one has $\mathrm{E}=\frac{1}{2} \mathrm{mv}^{2}$, in the relativistic case one obtains $\mathrm{E}=0$, since $\mathrm{L}$ is homogeneous. Since by construction $\mathbb{F L}$ is a presymplectic map, one finally has: A trajectory c on configuration space-time describes a free system iff $\left.\mathbb{F L} \circ \mathrm{c}^{\prime \prime}\right\lrcorner \omega=\mathrm{dH}$, where $\mathrm{H}=\mathrm{E}_{\bullet} \mathbb{F L} \mathrm{L}^{-1}$. Thus nonrelativistically $\mathrm{H}(\mathrm{p})=\frac{1}{2 \mathrm{~m}} \mathrm{k}(\mathrm{p}, \mathrm{p})$ and relativistically $\mathrm{H}=0 . \mathrm{H}$ is the free Hamiltonian of the system. If in the nonrelativistic case one replaces $\omega$ by $\omega_{0}=\omega+\mathrm{dH} \wedge \mathrm{dt}$ the Cartanform, then one obtains for relativistic as well as for nonrelativistic systems the fundamental principle of mechanical systems.

The Evolution space is a presymplectic manifold $\left(\mathrm{E}, \omega_{0}\right)$.

The trajectories of a free system are the integral manifolds of Ker $\omega$. For nonrelativistic systems the tyrajectories $c$ can be uniquely parametrized by $\left.c^{\prime}\right\lrcorner \mathbf{d t}=1$.

Examples: For a relativistic system one has $E=\left\{p \in T^{*} S \mid g^{*}(p, p)=m^{2}\right\}$ and $\omega_{0}$ is the restriction of the canonical symplectic form on the cotangent bundle to $\mathrm{E}$. $\mathrm{E}$ is a 7dimensional mass shell-bundle over the configuration space S.

Definition: A general mechanical system is a presymplectic manifold $\left(\mathrm{E}, \omega_{\mathrm{O}}\right)$. The integral manifolds of Ker $\omega$ are the trajectories of the free system.

The system is called Galilean iff there exists in addition a closed one form $\alpha$ on $\mathrm{E}$ with Ker $\alpha$ a transversal to Ker $\omega$. For $\alpha=d t$, $t$ is the time function of the system.

Forces: Definition: Forces are one forms $\mathrm{F} \in \mathrm{A}^{1} \mathrm{E}$.

Now Newton's law can be generalized to arbitrary evolution spaces: 
Newton's Law: The trajectories of a system with evolution space $\left(E, \omega_{0}\right)$ and force are given as reparametrizations of the vector field $\mathrm{X}$ on $\mathrm{E}$, where $\mathbf{X}\lrcorner \omega_{\mathrm{O}}=\mathbf{F}$.

Example: For a nonrelativistic particle system with evolution space $\mathbb{R} \times Q$, presymplectic form $\omega_{0}=+d h \wedge d t$, and force $F$, a curve parametrized by time $c(t)$ is a trajectory iff $\left.c^{\prime}(t)\right\lrcorner \omega+d h \wedge d t=F$. In this case one has in coordinates $(t, q, p)$ on $E c(t)=(t, q(t), p(t))$ and the Hamiltonian equations read:

$$
q^{\prime}(t)=\frac{p}{m} \text { and } p^{\prime}(t)=F
$$

The second equation is Newton's law in its original version.

A system is called conservative if $F=-$ gradV for some function $V: E \rightarrow \mathbb{R}$, the potential. For conservative systems the forces can be totally incorporated into the symplectic form:

Definition: For a conservative nonrelativistic system with evolution space $(E, \omega)$ and potential $\mathrm{V}: \mathrm{E} \rightarrow \mathbb{R}$ the presymplectic form $\omega_{\mathrm{V}}:=\omega_{\mathrm{O}}-\mathrm{dV} \wedge \mathrm{dt}$ is called the Cartan form of the system.

Proposition: The trajectories of a nonrelativistic system with conservative force $\mathrm{F}=-\mathrm{dV}$ are the integral manifolds of $\operatorname{Ker} \omega_{\mathrm{v}}$.

Comment: Thus for conservative systems the trajectories can be described in the same way as the trajectories of a free system, i.e. forces can be incorporated into the geometry of the system using the Cartanform.

This concept can also be applied to nonrelativistic quantum systems:

Definition: The evolution space $E$ of a quantum mechanical system is $E:=\mathbb{R} \times \mathbb{H}$ where $H$ is a complex separable Hilbert space. Let $\Omega$ be the presymplectic form induced by the imaginary part of the Hermitian product. Let $h: E \rightarrow \mathbb{R}$ be the expectation value of the free Schrödinger operator: $h(\phi)=<A \phi, \phi>$ with $A=i \Delta$. Then $\Omega+d h \wedge d t$ is a presymplectic form (it is only defined on a dense domain, but the geometric structure is unchanged) and the curves $\phi_{t}$ with $\phi_{t} \in \operatorname{Ker}(\Omega+d h \wedge d t)$ are the solutions of the free Schrödinger equation. Conservative forces are one forms of the form $\mathrm{dV}$, taken as multiplication operators in $\mathrm{E}$. 
Gauge theories: The first step in the program of incorporating nonconservative forces into geometry was performed by Einstein in general relativity. The geometrization of other forces other than gravity is the program of gauge theory, which was founded by Herman Weyl [14] and continued later by Kaluza-Klein, Yang-Mills and others. In the following it is shown how the Hamiltonian formalism for particle gauge theories can be seen as an example for general mechanical systems as defined above.

Let $\mathrm{G}$ be a Lie group with Lie algebra $\mathrm{LG}$ and $\mathrm{Z} \rightarrow \mathrm{M}$ a principal-G-bundle over a relativistic space-time (M.g). $Z$ represents an extended configuration space-time for a particle with inner degrees of freedom. In the case of a charged particle in an electromagnetic field, $\mathrm{G}$ is the circle $\mathrm{S}^{1}$ and for $\mathrm{G}=\mathrm{O}(3.1)$ one can recover the general relativistic description of a particle in gravity. Forces are represented as the curvature of a principal connection $\alpha \in \mathrm{A}^{1}(\mathrm{Z}, \mathrm{LG})$ on $\mathrm{P}$, while $\alpha$ plays the role of a potential and provides a decomposition $\mathrm{TP}=\mathrm{VerZ} \oplus$ HorZ. Assume in addition the existence of $\mathrm{G}$-invariant metric $\mathrm{h}$ on LG. Then, using the connection and the metric $\mathrm{g}$ on $\mathrm{M}$, one obtains a metric $\mathbf{k}: \mathrm{TZ} \times \mathrm{TZ} \rightarrow \mathbb{R}$ such that a $\mathrm{TP}=$ HorZ $\oplus$ VerZ is an orthogonal decomposition. Let $\mathrm{L}: \mathrm{TZ} \rightarrow \mathbb{R}$ be the norm \|\|$_{\text {of }} \mathbf{k}$.

The Legrendre transformation $\mathbb{F L}: \mathrm{TZ} \rightarrow \mathrm{T}^{*} \mathrm{Z}$ induces an immersion $\mathrm{PZ} \rightarrow \mathrm{T}^{*} \mathrm{P}$. If $\mathbf{k}^{*}$ denotes the induced dual metric on $\mathrm{T}^{*} \mathrm{P}$ then the image of $\mathbb{F L}$ is the $\mathbf{k}^{*}$-unitsphere in $\mathrm{T}^{*} \mathrm{P}$ and thus a presymplectic manifold. Since $\mathbf{k}$ is G-invariant $\mathbf{G}$ acts naturally and presymplecticly on image $\mathbb{F L}$. Reducing by $\mathrm{G}$ one obtains a presymplectic manifold $\mathrm{E} \subset \mathrm{T}^{*} \mathrm{M}$. The integral manifolds of the kernel of the presymplectic form on $\mathrm{E}$ are the trajectories of a particle in space-time moving under the influence of the force $F=c u r v \alpha$.

Example: For $\mathrm{G}=\mathrm{S}^{1}$ (the circle), the evolution space $\mathrm{E}$ will be the same as the evolution space of a free relativistic particle. But the symplectic structure will be $\omega_{0}+\tau^{*} \mathrm{~F}$, with $\tau: T^{*} M \rightarrow M$ the natural projection and $F=\operatorname{curv} \alpha \in A^{2}(M, \mathbb{R})$ since $G$ is abelian.

This construction can be generalized to configuration space-times of the form $\mathrm{P} \times \mathrm{Y}$, with $Y$ a symplectic homogeneous space of $G$ (see [3],[5],[8],[12],[13] for the symplectic construction).

These examples show that the notion of a general mechanical system is the natural frame to describe mechanical systems, relatisicic or nonrelativistic, classical or quantum in 
the spirit of Einstein and Weyl as a geometric reduction to the free case. Though generalized to nonlinear spaces, the basic principle is modified by the notion of relativity and expressed in the language of modern abstract geomery, still the same: GALILEI'S PRINCIPLE, the basis of modern physics.

\section{REFERENCES:}

[1] R.Abraham/J.E.Marsden: Foundations of Mechanics (Benjamin/Cummings 1987

[2] D.Bleeker: Gauge Theory and Variational Principles (Addison-Wesley 1981)

[3] W.D.Curtis/F.R.Miller: Differential Manifolds and Theoretical Physics (Academic Press 1985)

[4] J.Ehlers/F.A.E.Pirani/A.Schild: The Geometry of Free Fall and Light propagation in "General Relativiry" (ed. L. O'Raifeartaigh) (1972)

[5] V.Guillemin/S.Sternberg: Symplectic techniques in physics (Cambridge Univ.Press 1984)

[6] C.Günther: Logic, Dynamics, Symmetry of Particle Systems Winter Research Inst.Geom.Quantization, (M.Gotay Ed.) (1981)

[7] J.E.Marsden: Hamiltonian One Parameter Groups Arch.Rat.Mech.Anal.28,362-396 (1968)

[8] R.Montgomery:Canonical Formulations of a Classical Particle in a Yang-Mills Field and Wongs Equations. Lett.Math.Phys.8, 59-67 (1984)

[9] R.S.Palais: The Geometrization of Physics, Lect.Notes in Math. Nat.Tsing Hua University (1981)

[10] H.Rund: The Hamilton-Jacobi theory in the calculus of variations (van Nostrand 1966)

[11] J.M. Souriau: Structure des systèmes dynamiques (Dunod 1970)

[12] S.Sternberg: Minimal coupling and the symplectic mechanics of a classical particle in the presence of a Yang-Mills field.Proc.Nat.Acad.Sci.USA,24, 5253- 5254 (1977) 
[13] A.Weinstein: A Universal Pahse Space for Particles in Yang-Mills Fields. Lett.Math.Phys. 2, 417-420 (1978)

[14] H.Weyl: Space-Time-Matter (Dover 1950) 


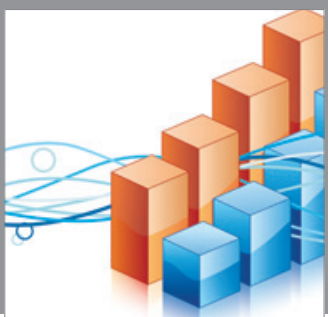

Advances in

Operations Research

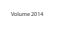

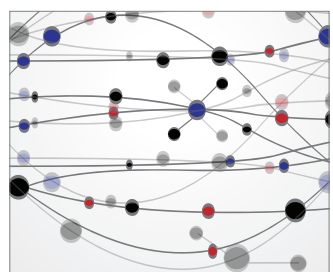

\section{The Scientific} World Journal
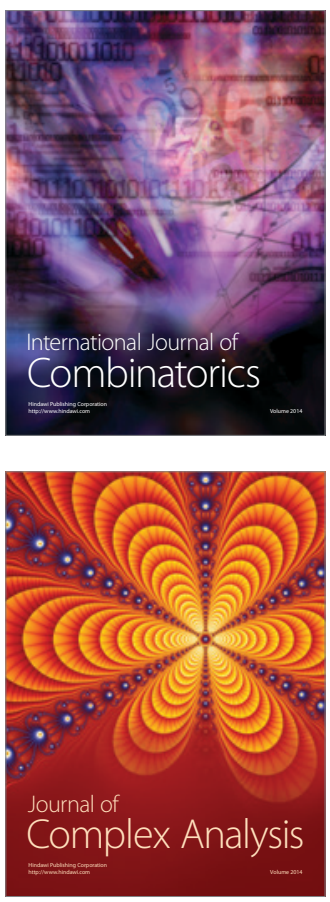

International Journal of

Mathematics and

Mathematical

Sciences
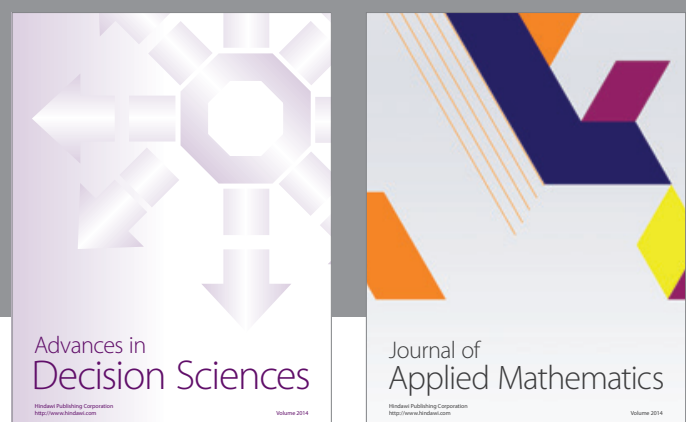

Journal of

Applied Mathematics
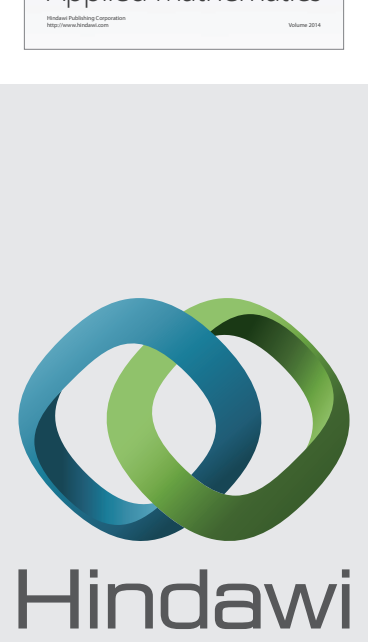

Submit your manuscripts at http://www.hindawi.com
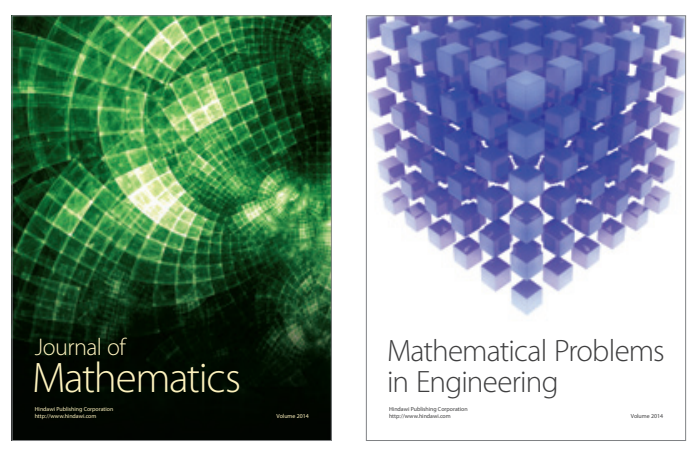

Mathematical Problems in Engineering
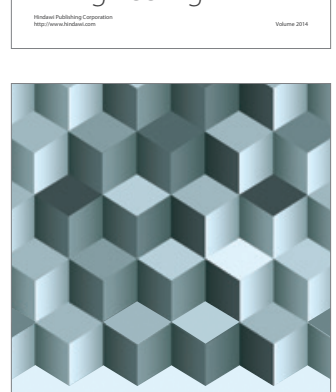

Journal of

Function Spaces
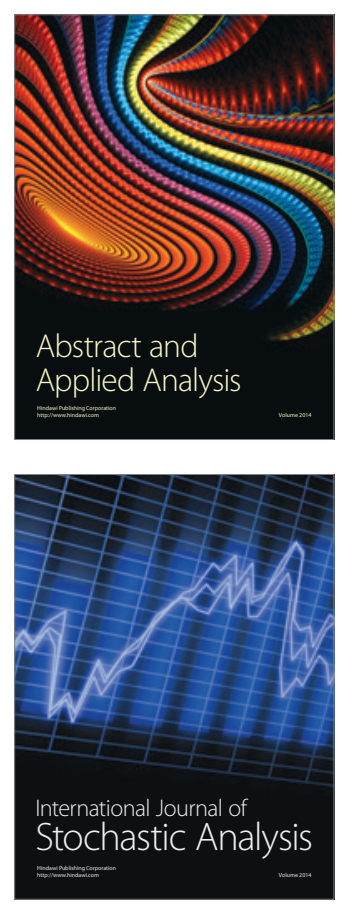

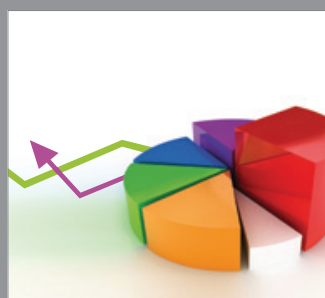

ournal of

Probability and Statistics

Promensencen
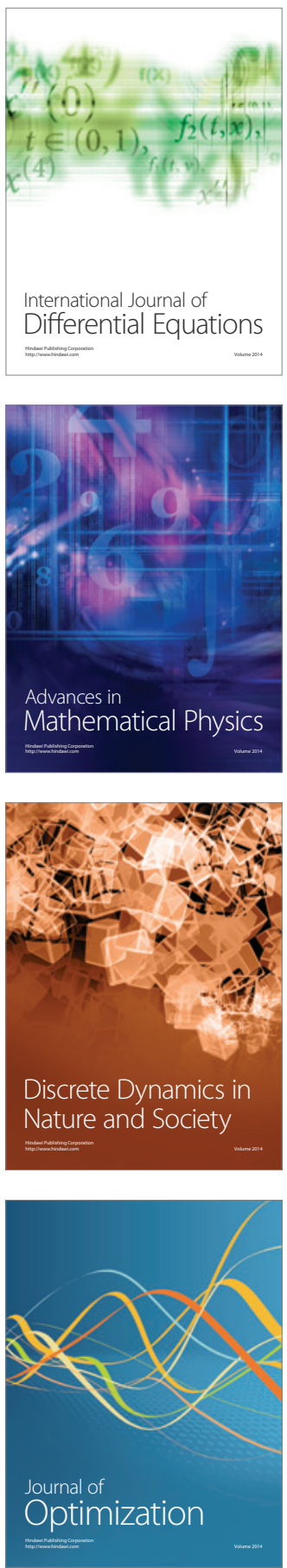